\title{
Ultrasensitive iron-based magnetic resonance contrast agent constructed with natural polyphenol tannic acid for tumor theranostics
}

\author{
Lu An, Yu Cai, Qiwei Tian*, Jiaomin Lin and Shiping Yang*
}

\begin{abstract}
Because of the toxicity of Gd(III) complexes and the poor $T_{1}$ magnetic resonance imaging (MRI) contrast of superparamagnetic iron oxide, the development of new stable, non-toxic, and efficient contrast agents is desirable. Herein, tannic acid (TA), a large natural polyphenol, and bovine serum albumin (BSA) were used to construct non-toxic Fe(III) complexes with increased relaxivity based on a strategy slowing the molecular spin. Compared with the commercial $T_{1}$ contrast agent Magnevist ${ }^{\circledR}$, TA-Fe@BSA not only exhibits comparable $T_{1}$ MRI contrast enhancement under $0.5,1$ and $7 \mathrm{~T}$ magnetic fields both in vitro and in vivo, but also has better stability and biocompatibility. Moreover, TA-Fe@BSA with near-infrared (NIR) absorption demonstrates efficient tumor ablation via photothermal effects. These results demonstrate their strong potential as an alternative $T_{1}$ MRI contrast agent and tumor theranostics agent in clinical settings.
\end{abstract}

Keywords: tannic acid, Fe(III) complex, low-toxic, MRI contrast agent, photothermal therapy

\section{INTRODUCTION}

Magnetic resonance imaging (MRI) has been widely used in medical diagnosis due to its advantages of non-invasiveness, non-radiativity and high spatial resolution, especially for the examination of soft tissue lesions $[1,2]$. The enhanced $T_{1}$ contrast is favored because it improves imaging, avoids the "blooming effect", and makes it easier to distinguish between different regions [3,4]. Among the reported $T_{1}$ contrast agents, $\mathrm{Gd}(\mathrm{III})$-based complexes are already in clinical use [5-7], and superparamagnetic iron oxide nanoparticles (NPs) (SPIONs), which exhibit $T_{1}$ contrast at sizes $<3 \mathrm{~nm}$, are the most promising for clinical use because of their good biological compatibility $[8,9]$. However, they both have some intrinsic flaws, including the renal toxicity of $\mathrm{Gd}$, and the easy aggregation of SPIONs in vivo, which result in poor $T_{1}$ contrast because of the loss of their ultra-small size $[10,11]$. Therefore, it is of great practical significance to develop a new $T_{1}$ contrast agent with two advantages of (1) low toxicity and (2) efficient contrast performance.

The twisted octahedral geometry of $\mathrm{Fe}(\mathrm{III})$ containing weak-field ligands results in high-spin $\mathrm{d}^{5}$ complexes, which has properties similar to clinically applied Gd(III) agents $[12,13]$. More importantly, iron as an endogenous metal, its complexes exhibit lower cytotoxicity and have been proposed as an alternative to $\mathrm{Gd}(\mathrm{III})$ complexes $[14,15]$. Gallic acid-Fe coordination polymers with lower biotoxicity have been reported as $T_{1}$ contrast agents by our group [16] and Liu et al. [17]. However, the imaging performances of these contrast agents are not very satisfactory due to the low relaxivity, and there are few indepth studies on their toxicity and applicability in vivo. Hence, a significant amount of work still needs to be carried out to replace Gd(III) agent. To improve the imaging performance and relaxivity, the inner-sphere and outer-sphere relaxation mechanisms need to be considered. The inner-sphere mechanism involves three key factors, the spin correlation time of the compound $\left(\tau_{\mathrm{R}}\right)$, the residence lifetime of inner bound water $\left(\tau_{\mathrm{m}}\right)$, and the number of water molecules directly coordinated to the metal ion $(q)$; while the diffusion correlation time $\left(\tau_{\mathrm{D}}\right)$ of water molecules determines relaxivity based on the outersphere mechanism $[18,19]$. The increase in relaxivity can be achieved by increasing $\tau_{\mathrm{R}}, \tau_{\mathrm{D}}, q$ and $1 / \tau_{\mathrm{m}}$. Another key factor to be considered is the toxicity and stability.

The Key Laboratory of Resource Chemistry of the Ministry of Education, Shanghai Key Laboratory of Rare Earth Functional Materials, and Shanghai Municipal Education Committee Key Laboratory of Molecular Imaging Probes and Sensors, Shanghai Normal University, Shanghai 200234, China

* Corresponding authors (emails: shipingy@shnu.edu.cn (Yang S); qiweitian@shnu.edu.cn (Tian Q)) 
Therefore, choosing a appropriate ligand to increase the relaxivity while reducing the toxicity of the Fe complexes is a promising way.

Herein, as a proof of concept, natural polyphenolic tannic acid (TA), bovine serum albumin (BSA) and $\mathrm{Fe}$ (III) salt were used as safe materials for the construction of Fe complex (TA-Fe@BSA). TA, which is widely used in the pharmaceutical, food additive and biomedical fields because of its non-toxic and antibacterial properties [20,21], has been demonstrated to form a highly stable complex with $\mathrm{Fe}[22,23]$. More importantly, the molecular structure of TA is larger compared with gallic acid ligand, which is beneficial to slowing down the molecular spin and increasing $\tau_{\mathrm{R}}$ after coordinate with Fe [24,25]. BSA, which is biodegradable and non-immunogenic [2628], not only improves biocompatibility but also restricts the rotation of $\mathrm{Fe}$ and the diffusion of water molecules because of the macromolecular steric effects [29]. This can effectively increase $\tau_{\mathrm{R}}$ and $\tau_{\mathrm{D}}$, further improving the MRI contrast performance for in vivo imaging (Scheme 1). In addition, $\mathrm{TA}-\mathrm{Fe}^{3+}$ coordination complex has high potential for tumor photothermal therapy (PTT) [30]. As expected, the obtained TA-Fe@BSA not only had good stability and biocompatibility, but also showed excellent MRI contrast enhancement under magnetic fields of 0.5 , 1 and $7 \mathrm{~T}$. The relaxivity was comparable to that of the commercial contrast agent Gd(DTPA) (Magnevist). Moreover, TA-Fe@BSA can be applied for efficient tumor ablation as a photothermal agent due to the absorption in the near-infrared (NIR) regions. This study proposes a process to develop low-toxicity Fe(III)-based complexes with enhanced MRI performance and PTT effects.

\section{EXPERIMENTAL SECTION}

\section{Materials and characterization}

TA (99\%) and $\mathrm{FeCl}_{3} \cdot 6 \mathrm{H}_{2} \mathrm{O}$ were purchased from Sinopsin chemical reagents (Shanghai). BSA was purchased from Amresco (USA). All reagents were used directly without further purification. A JEOL jem-2011f transmission electron microscope (TEM, Japan) was used to characterize the morphology of the obtained NPs. The size of the obtained NPs was determined via atomic force microscope (AFM, Bruke NanoScope IIIa SPM, German) and dynamic light scattering measurements (DLS, Malvern nano-zs90, UK). A Fourier-transform infrared spectrophotometer (Nicolet Avatar 370 FT-IR spectrophotometer) was used to measure the composition of the obtained NPs using the potassium bromide tablet method. The absorption spectra of the obtained NPs were measured on a UV-Vis-NIR spectrophotometer (Beckman Coulter DU 730, USA). The Fe content was confirmed by using an inductively coupled plasma atomic emission spectrometer (ICP-AES, Varian VistaMPX, USA).

\section{Synthesis of TA-Fe@BSA NPs}

The BSA-Fe complex was prepared by mixing the BSA solution $\left(10 \mathrm{~mL}, 2.22 \mathrm{~g} \mathrm{~mL}^{-1}\right)$ and $\mathrm{FeCl}_{3}$ aqueous solution $\left(108 \mu \mathrm{L}, \quad 0.1 \mathrm{~g} \mathrm{~mL}^{-1}\right)$ under magnetic stirring. After $30 \mathrm{~min}, 1.02 \mathrm{~mL}$ of $\mathrm{TA}$ aqueous solution $\left(10 \mathrm{mg} \mathrm{mL}^{-1}\right)$ was added dropwise to the formed BSA-Fe complex so-

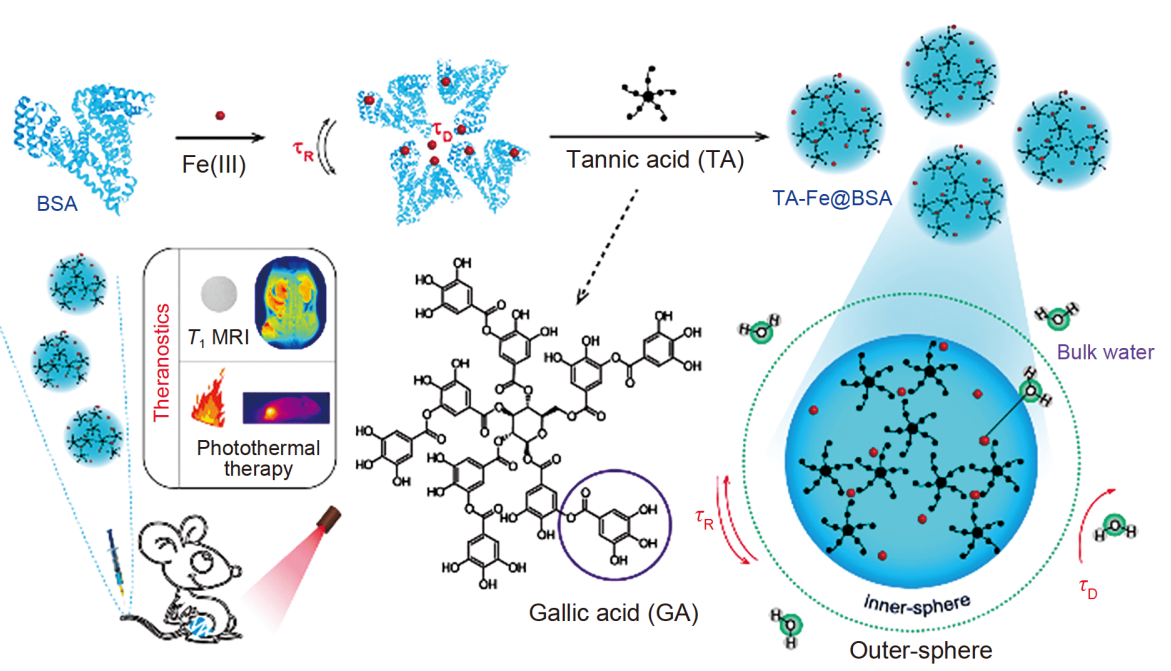

Scheme 1 Schematic illustration of the fabrication of TA-Fe@BSA, and the key factors that affect relaxivity, as well as their application in $T_{1}$ MRI and PTT. 
lution under stirring. The mixture was stirred for $19 \mathrm{~h}$ at room temperature. Then, the supernatant was collected via centrifugation at $8000 \mathrm{rmin}^{-1}$. Finally, the TA-Fe@ BSA NPs were obtained from the collected supernatant via ultrafiltration centrifugation at $3500 \mathrm{r} \mathrm{min}^{-1}$ for $5 \mathrm{~min}$ with a molecular weight cut-off of $100 \mathrm{kDa}$.

\section{In vitro $\mathrm{MRI}$}

To study the MRI properties of the TA-Fe@BSA NPs, an aqueous solution containing different concentrations of Fe $\left(0,0.125,0.25,0.5,1\right.$ and $\left.2 \mathrm{mmol} \mathrm{L}^{-1}\right)$ was placed in a 0.5 T MRI system (NMI20-Analyst; Niumag) to test the longitudinal relaxation time $\left(T_{1}\right)$ and transverse relaxation time $\left(T_{2}\right) . T_{1}$-weighted and $T_{2}$-weighted MRIs for TA-Fe@BSA NPs were also taken. $T_{1}$ was measured using a hard-pulse Q-IR sequence. $T_{2}$ was measured by a hardpulse CPMG sequence. $T_{1}$ and $T_{1}$-weighted MR imaging were also tested under $1 \mathrm{~T}$ (NMG1-Analyst) and $7 \mathrm{~T}$ (Bruke BioSpec70/20 USR) magnetic fields.

\section{Biocompatibility}

Mouse breast cancer 4T1 cells and human umbilical vein endothelial cells (HUVEC) were used to evaluate the cytotoxicity of the TA-Fe@BSA NPs. The cells were placed in a 96-well plate and cultured for $12 \mathrm{~h}$ in a cell incubator $\left(37^{\circ} \mathrm{C}, 5 \% \mathrm{CO}_{2}\right)$. As the cells grew into the logarithmic phase, various concentrations of NPs $(0,0.06,0.12,0.25$ and $0.5 \mathrm{mmol} \mathrm{L}^{-1}$ ) were added. After 12 and $24 \mathrm{~h}$ of incubation, the cell viability was investigated using a 3-(4,5dimethyl-2-thiazolyl)-2,5-diphenyl-2-H-tetrazolium bromide (MTT) assay. In addition, the $4 \mathrm{~T} 1$ cells were incubated with different concentrations $\left(0-6 \mathrm{mmol} \mathrm{L}^{-1} \mathrm{Fe}\right)$ of NPs for $24 \mathrm{~h}$, and the IC50 value was calculated using GraphPad Prism 6 with six parallel samples per concentration.

Hemolysis assays were carried out as follow: $0.4 \mathrm{~mL}$ of red blood cells (RBCs, $2 \%$ volume ratio) were treated with $1 \mathrm{~mL}$ of deionized water, phosphate buffered saline (PBS), or PBS containing TA-Fe@BSA NPs $(60,120,250$, or $\left.500 \mu \mathrm{mol} \mathrm{L}^{-1}\right)$. After $1 \mathrm{~h}$, the mixtures were centrifuged to collect the supernatant. The hemolysis ratio was tested using the absorbance of the supernatant at $570 \mathrm{~nm}$ and calculated by

Hemolysis ratio $=\frac{A_{\text {sample }}-A_{\mathrm{PBS}}}{A_{\mathrm{H}_{2} \mathrm{O}}-A_{\mathrm{PBS}}} \times 100 \%$.

After intravenous injection of the TA-Fe@BSA NPs $\left(2 \mathrm{mmol} \mathrm{L}^{-1}, 100 \mu \mathrm{L}\right)$ for 16 days, mouse blood was collected for biochemical index analysis, including alkaline phosphatase (ALP), alanine aminotransferase (ALT), as- partate aminotransferase (AST) for liver function evaluation, creatinine (CREA), and blood urea nitrogen (BUN) for renal function evaluation. For the blood routine examination, the white blood cell count (WBC), $\mathrm{RBC}$, mean cell hemoglobin $(\mathrm{MCH})$, mean cell volume (MCV), hematocrit (HCT), hemoglobin concentration (HGB), mean corpuscular hemoglobin concentration (MCHC), and platelets (PLT) were determined. In addition, the main organs including the heart, liver, spleen, lung and kidney were collected, and the damage to the organs by TA-Fe@BSA was evaluated from the H\&E stained slices. The healthy mice treated with PBS $(100 \mu \mathrm{L})$ were set as control $(n=3)$.

\section{In vivo MRI studies}

In vivo MRI studies were carried out using the following equipment: $0.5 \mathrm{~T}$ (Niumag MiniMR-60), $1 \mathrm{~T}$ (NMG1Analyst), and $7 \mathrm{~T}$ (Bruke BioSpec70/20 USR). Magnevist and TA-Fe@BSA NPs $\left(20 \mu \mathrm{L}, 2 \mathrm{mmol} \mathrm{L}^{-1}\right)$ were injected intratumorally into symmetrical sides of the $4 \mathrm{~T} 1$ bilateral tumor-bearing mice, respectively. Cross-sectional MRI scans were performed before and $0.5 \mathrm{~h}$ after intratumoral injection of TA-Fe@BSA NPs.

After intravenous injection of TA-Fe@BSA NPs $\left(150 \mu \mathrm{L}, 2 \mathrm{mmol} \mathrm{L}^{-1}\right)$, coronal plane scanning was performed before and after NP injections $(0.5,1,2,4$ and $6 \mathrm{~h})$.

\section{The biodistribution analysis}

To determine the metabolism pathway of TA-Fe@BSA, the $4 \mathrm{~T} 1$ tumor-bearing mice were randomly divided into six groups $(n=3)$, and received intravenous injection with TA-Fe@BSA NPs $\left(150 \mu \mathrm{L}, 2 \mathrm{mmol} \mathrm{L}^{-1}\right)$ at different time points $(0.5,1,2,4,6$ and $24 \mathrm{~h})$, respectively. After that, the mice were sacrificed, and the main organs and metabolites (heart, liver, spleen, lung, kidney, tumor, urine and feces) were collected for ICP-AES analysis of Fe content. The distribution of TA-Fe@BSA NPs was calculated as the injected dose percentage per gram of tissue (\% ID/g).

\section{Measurement of photothermal performance}

The temperature change of the TA-Fe@BSA NPs with different Fe concentrations (0.03, 0.06, 0.12, 0.25, 0.5, 1 and $1.5 \mathrm{mmol} \mathrm{L}^{-1}$ ) was collected using an FLIR A300 thermal camera with irradiation by a $808 \mathrm{~nm}$ laser $\left(1 \mathrm{~W} \mathrm{~cm}^{-2}\right)$ for $15 \mathrm{~min}$. Water was used as control. The photostability was measured by the absorption change of the NPs before and after irradiation. For the temperature change, the NPs were treated with eight cycles of $15 \mathrm{~min}$ 
laser exposure followed by $15 \mathrm{~min}$ rest.

\section{Photothermal ablation of tumor}

Four groups, including PBS, PBS+Laser, TA-Fe@BSA NPs, and TA-Fe@BSA NPs+Laser, were used to evaluate the photothermal effects of TA-Fe@BSA NPs both in vitro and in vivo. The laser wavelength was $808 \mathrm{~nm}$ with a power density of $1 \mathrm{~W} \mathrm{~cm}^{-2}$, and the irradiation time was 5 min. PBS, PBS+Laser, and TA-Fe@BSA NPs $\left(1 \mathrm{mmol} \mathrm{L}^{-1}\right)$ were the control, laser control, and agent control groups, respectively, while the TA-Fe@BSA NPs $\left(1 \mathrm{mmol} \mathrm{L}^{-1}\right)+$ Laser group was the treatment group.

For the in vitro investigation, the treatment effect was evaluated via trypan blue staining. The cells in a 96-well plate were divided into the above four groups. PBS was added into the wells for the groups of PBS and PBS +Laser, while $1 \mathrm{mmol} \mathrm{L}^{-1}$ TA-Fe@BSA NPs was used for the groups of TA-Fe@BSA NPs and TA-Fe@BSA NPs + Laser. Experiments on the four groups were carried out in parallel. After exposing the cells to the laser for the groups of PBS+Laser and TA-Fe@BSA NPs+Laser, all the cells in the four groups were stained with trypan blue for 15 min. Then, the cells were washed with PBS and observed using an inverted optical microscope (Motic AE2000).

For in vivo investigation, $4 \mathrm{~T} 1$ tumor-bearing mice were used as the tumor model and randomly divided into the above four groups, with six mice in each group. For the groups of PBS and TA-Fe@BSA NPs, the mice were only intravenously injected with PBS $(20 \mu \mathrm{L})$ and TA-Fe@BSA NPs $\left(20 \mu \mathrm{L}, 2 \mathrm{mmol} \mathrm{L}^{-1}\right)$, respectively. The mice in the groups of PBS+Laser and Fe@BSA NPs+Laser were exposed to the laser $2 \mathrm{~h}$ after injection with PBS $(20 \mu \mathrm{L})$ and TA-Fe@BSA NPs $\left(20 \mu \mathrm{L}, 2 \mathrm{mmol} \mathrm{L}^{-1}\right)$, respectively. Both the injection and laser irradiation were only given on the first day. After laser irradiation, each group receiving laser treatment was imaged and their temperatures were monitored by using an NIR thermal imaging camera.

For histopathological section analysis, one mouse from each group was sacrificed after treatment and the tumor was excised, and hematoxylin eosin (H\&E) staining and terminal deoxynucleotidyl transferase (TdT)-mediated 2'deoxyuridine $5^{\prime}$-triphosphate (dUTP) nick end labeling (TUNEL) were used to evaluate the necrosis and apoptosis rate of the tumor. The tumor size and body weight of the remaining mice were recorded every day for 16 consecutive days.

\section{Ethical statement}

All animal experiments were carried out in strict ac- cordance with the guidelines for care and use of laboratory animals of Shanghai Normal University and approved by the Animal Ethics Committee of Shanghai Normal University.

\section{RESULTS AND DISCUSSION}

The designed TA-Fe@BSA complex was synthesized using a modified version of our previous report [16]. TA was added dropwise into the mixture of $\mathrm{Fe}$ (III) and BSA to form a Fe complex, where TA coordinated with Fe(III) via the phenolic hydroxyl group. During the growth processes, BSA not only stabilized the NPs by serving as a carrier of $\mathrm{Fe}(\mathrm{III})$ via coordination of the N-terminal amines and cysteine residues with $\mathrm{Fe}(\mathrm{III})$, but also improved the biocompatibility by virtue of its biodegradable and non-immunogenic features [26,27]. The relationship between the Fe(III):TA molar ratio and the relaxivity was first explored (Table S1). The relaxivity of TA-Fe@BSA increased as TA increased until a molar ratio of 1:0.15 $\left(r_{1}\right.$ $\left.=4.20, r_{2}=4.49\right)$. As the TA concentration continued to increase, rapid precipitation was observed at molar ratios of 1:0.5 and 1:1. Thus, TA-Fe@BSA synthesized at this condition (Fe(III):TA = 1:0.15) was selected for further investigation. TEM, AFM and DLS were used to determine the morphology and size distribution of the obtained TA-Fe@BSA. As expected, monodisperse, spherical nanodots with an average size of $\sim 21 \mathrm{~nm}$ were successfully prepared (Fig. 1a, b and Fig. S1). The hydrodynamic diameter increased to $(28.8 \pm 3.7) \mathrm{nm}$, which could be ascribed to the hydrophilicity of the outer BSA layer (Fig. 1c) [31]. In addition, the peaks at 1206 and $1085 \mathrm{~cm}^{-1}$ in the FT-IR spectra could be assigned to TA, the 1647 and $1534 \mathrm{~cm}^{-1}$ peaks were characteristics of the amide I and amide II band vibrations of BSA (Fig. 1d) [30,32]. The slight shift indicates the coordination of $\mathrm{Fe}$ (III) and TA and also the interaction between Fe(III) and the amide of BSA. This was further confirmed by the VisNIR absorption spectra and fluorescence spectra. An obvious ligand-to-metal charge-transfer (LMCT) band could be observed at $570 \mathrm{~nm}$ for TA-Fe@BSA because of $\mathrm{TA}$ and $\mathrm{Fe}$ (III) coordination (Fig. 1e) [22], and the fluorescence of BSA was quenched because of the combination of Fe(III) and BSA (Fig. S2). Furthermore, the NPs maintained proper size and absorption stability (Fig. If and Fig. S3). The above results all indicate the successful synthesis of TA-Fe@BSA.

To determine whether TA-Fe@BSA could serve as an MRI contrast agent, longitudinal $\left(T_{1}\right)$ weighted and transverse $\left(T_{2}\right)$ weighted MRI and the corresponding relaxivities were evaluated on a $0.5 \mathrm{~T}$ MRI scanner. GA- 

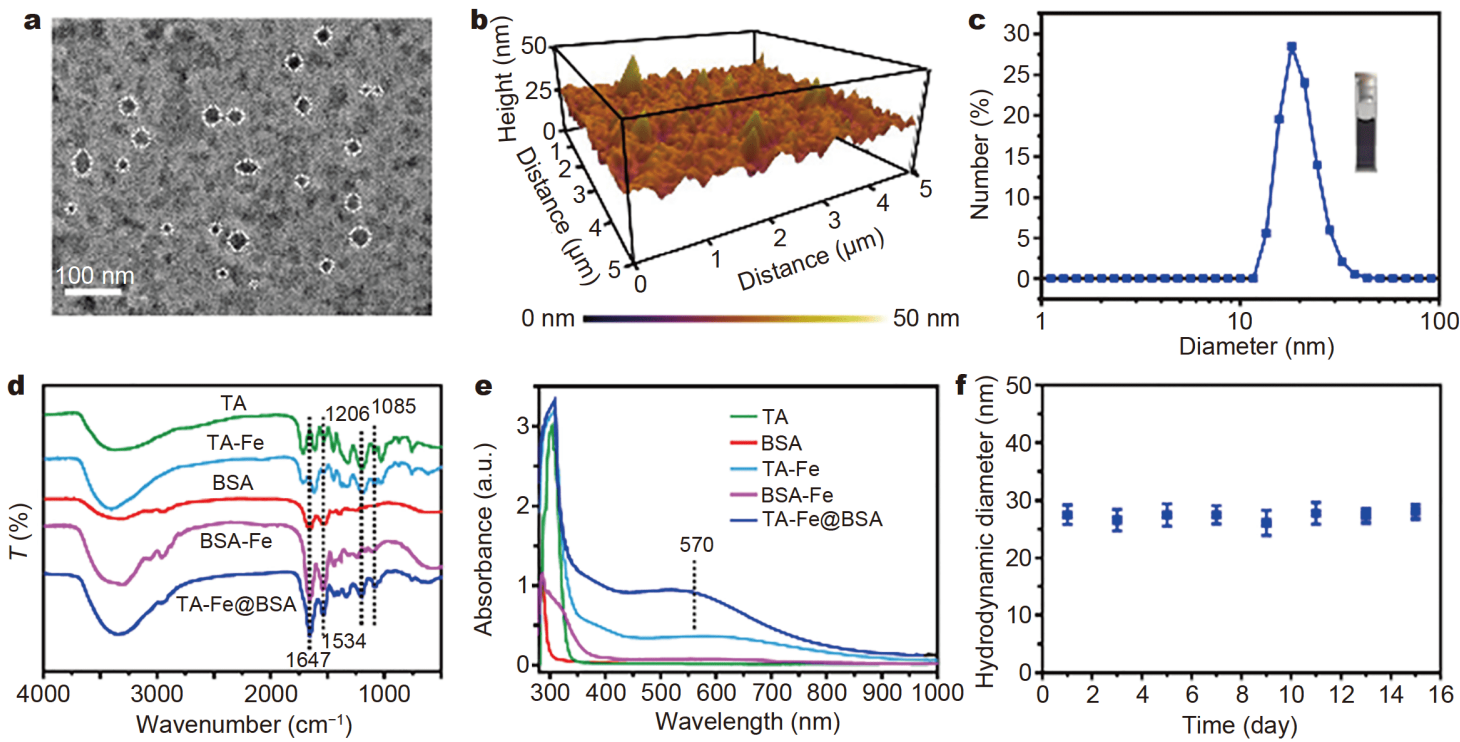

Figure 1 Synthesis and characterization of TA-Fe@BSA. (a) TEM image. Some of NPs are highlighted by the white circles. (b) 3D AFM topography of TA-Fe@BSA. (c) Hydrodynamic size distribution, and photograph of the solution. (d) FT-IR spectra and (e) Vis-NIR spectra of TA, BSA, TA-Fe, BSA-Fe and TA-Fe@BSA. (f) Hydrodynamic diameter tracking of TA-Fe@BSA.

Fe@BSA and Magnevist were used as the control and measured with the same parameters. As the concentration of Fe or Gd increased, the $T_{1}$-weighted images in all three groups gradually brightened (Fig. 2a). The MRI performance of TA-Fe@BSA approached that of Magnevist at high concentrations, and it performed significantly better than GA-Fe@BSA. None of the three groups showed visible $T_{2}$-weighted imaging. The longitudinal relaxivity $\left(r_{1}\right)$ of TA-Fe@BSA was $4.20 \mathrm{~L} \mathrm{mmol}^{-1} \mathrm{~s}^{-1}$, which was close to that of Magnevist $\left(4.71 \mathrm{~L} \mathrm{mmol}^{-1} \mathrm{~s}^{-1}\right)$ and much higher than that of GA-Fe@BSA $\left(0.79 \mathrm{~L} \mathrm{mmol}^{-1} \mathrm{~s}^{-1}\right)$. This is because of the slow spin dynamics of the compound, which can be attributed to its large structure. Moreover, the $r_{2} / r_{1}$ of TA-Fe@BSA was 1.06, lower than those of Magnevist (1.12) and GA-Fe@BSA (1.10), which is more beneficial to $T_{1}$ MRI (Fig. 2b). Similarly, because the spatial limiting effect of BSA [29,33], the $r_{1}$ of TAFe@BSA was also higher than that of TA-Fe $\left(2.08 \mathrm{~L} \mathrm{mmol}^{-1} \mathrm{~s}^{-1}\right.$, Fig. S4), which demonstrates that TA-Fe@BSA has potential as a positive $T_{1}$ MRI contrast agent. Motivated by the high MRI contrast at low fields, $T_{1}$-weighted imaging was further explored at higher fields ( 1 and $7 \mathrm{~T}$ ). A similar brightening trend for $0.5 \mathrm{~T}$ was observed with increasing concentration. An enhanced field strength resulted in clearer imaging (Fig. $2 \mathrm{c}$ and d). The $r_{1}$ of TA-Fe@BSA, Magnevist and GA-Fe@BSA were 3.47, 3.89 and $0.86 \mathrm{~L} \mathrm{mmol}^{-1} \mathrm{~s}^{-1}$ at $1 \mathrm{~T}$, respectively, while they were $1.10,1.27$ and $0.13 \mathrm{~L} \mathrm{mmol}^{-1} \mathrm{~s}^{-1}$ at $7 \mathrm{~T}$, respectively (Fig. 2e). The decrease of $r_{1}$ with increasing magnetic field strength could be attributed to the increase in the proton Larmor frequency, which exceeds the frequency of molecular vibration, thereby interfering with the relaxation of spin-lattice $[29,34]$. Overall, the enhanced MRI contrast and significantly improved $r_{1}$ were comparable to that of commercial Magnevist; hence, TAFe@BSA has potential for use as a $T_{1}$-weighted MRI contrast agent.

In addition to the contrast performance, good biocompatibility is a key prerequisite for practical application of an MRI contrast agent. The biocompatibility of TA-Fe@BSA was demonstrated via cytotoxicity tests, hemolysis assays, blood routine tests, and analysis of the biochemical indexes in mice, along with the analysis of H\&E stained major organs' slices. TA-Fe@BSA displayed no apparent toxicity to $4 \mathrm{~T} 1$ and HUVEC cell lines, even at Fe concentrations up to $1 \mathrm{mmol} \mathrm{L}^{-1}$ (Fig. 3a). Furthermore, the IC50 value for $4 \mathrm{~T} 1$ cells was $3.31 \pm 0.24 \mathrm{mmol} \mathrm{L}^{-1}$ (Fig. $3 \mathrm{~b}$ and Fig. S5), higher than the safe dose of Magnevist [35], and the hemolysis rate of RBCs was as low as $0.36 \%$, indicating that the safety of TA-Fe@BSA (Fig. 3c). The in vivo toxicity was further assessed after intravenous injection of TA-Fe@BSA. Compared with healthy mice, there was no significant difference in liver and kidney function and for the blood routine indicators of the tested mice (Fig. 3d). No inflammatory infiltration and injury were observed in the 
a
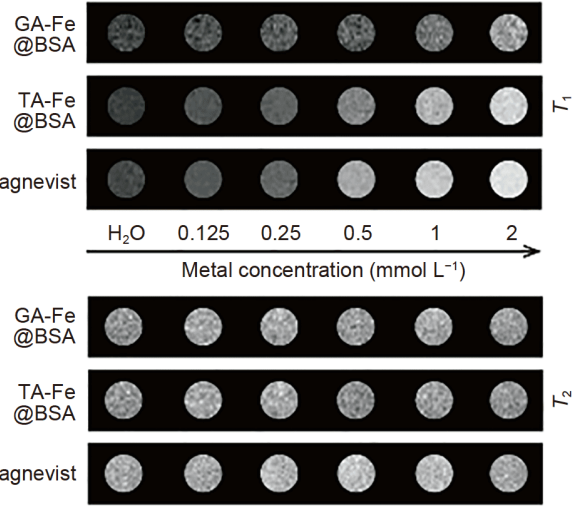

$\mathbf{C}$

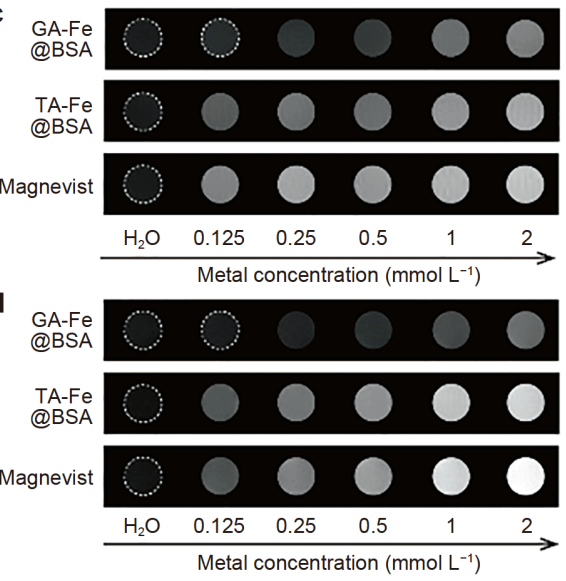

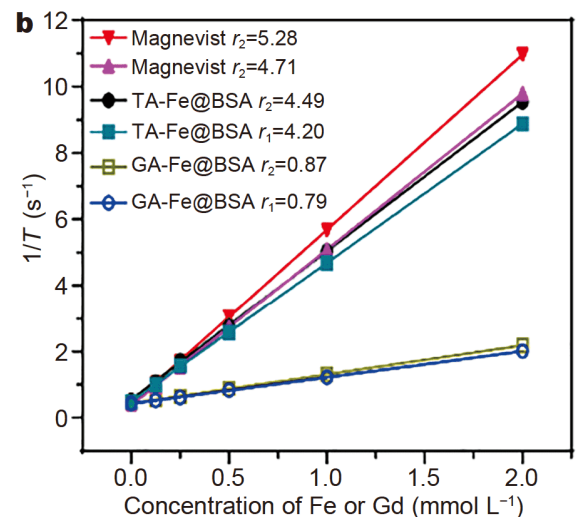

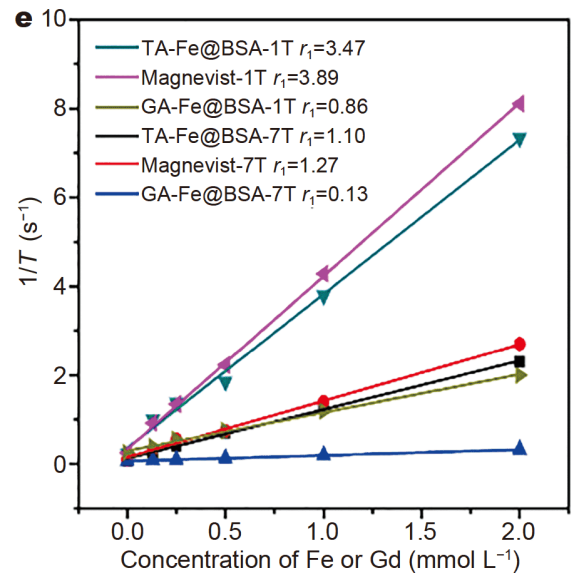

Figure 2 (a) $T_{1}$-weighted and $T_{2}$-weighted MRI scans $(0.5 \mathrm{~T})$ and (b) corresponding relaxivity fitting curves of GA-Fe@BSA, TA-Fe@BSA and Magnevist. $T_{1}$-weighted MR images of TA-Fe@BSA compared with GA-Fe@BSA and Magnevist at (c) 1 T and (d) 7 T. (e) Corresponding relaxivity fitting curves of GA-Fe@BSA, TA-Fe@BSA and Magnevist at 1 and 7 T.

major organs either (Fig. 3e). All the data indicated that TA-Fe@BSA was safe, non-toxic, and had good biocompatibility.

The in vivo $T_{1}$-weighted MRI performance was first studied with a $0.5 \mathrm{~T}$ MRI scanner. TA-Fe@BSA and Magnevist were intratumorally injected into the symmetrical sides of different bilateral tumor-bearing mice. Similarly to the results in solution, TA-Fe@BSA exhibited contrast enhancement close to commercial Magnevist, and they both had significantly increased signal intensity (Fig. 4a and b) [36]. Next, after intravenous injection of TA-Fe@BSA, the $T_{1}$-weighted MRI scans of the 4T1 tumor-bearing mice were monitored. The contrast of the main organs, including the tumor, kidney, liver and bladder was gradually enhanced with time, and the maximum MRI signal intensity appeared $\sim 2 \mathrm{~h}$ post-injection (Fig. 4c and d). More importantly, the signal intensity of the tumor site obviously increased compared with the pre-injection intensity, indicating that TA-
Fe@BSA can be used for MRI of tumors.

To further validate the viability of TA-Fe@BSA, in vivo MRI at higher field strengths ( 1 and $7 \mathrm{~T}$ ) was carried out after intravenous injection. As expected, they presented positive MRI contrast enhancement with a higher resolution as the field strength increased. After $\sim 1-2 \mathrm{~h}$ post-injection, the tumor site, liver and kidney showed the strongest $T_{1}$ contrast effect at $1 \mathrm{~T}$, while the bladder could be observed at $\sim 2-4 \mathrm{~h}$, which could be ascribed to the metabolic processes (Fig. 5a). The MRI trend was similar with a $7 \mathrm{~T}$ field, except for the bladder (Fig. 5b). The inconsistencies may be because of the individual differences between the mice. Overall, the imaging time was much longer than elimination half-life of commercial Magnevist [37], which is favorable for long-term observation and accurate localization of the lesions. The imaging changes can be seen more clearly by the MRI signal intensity. The signal value in most organs increased over time until it reached the maximum at $\sim 1-2 \mathrm{~h}$, and 

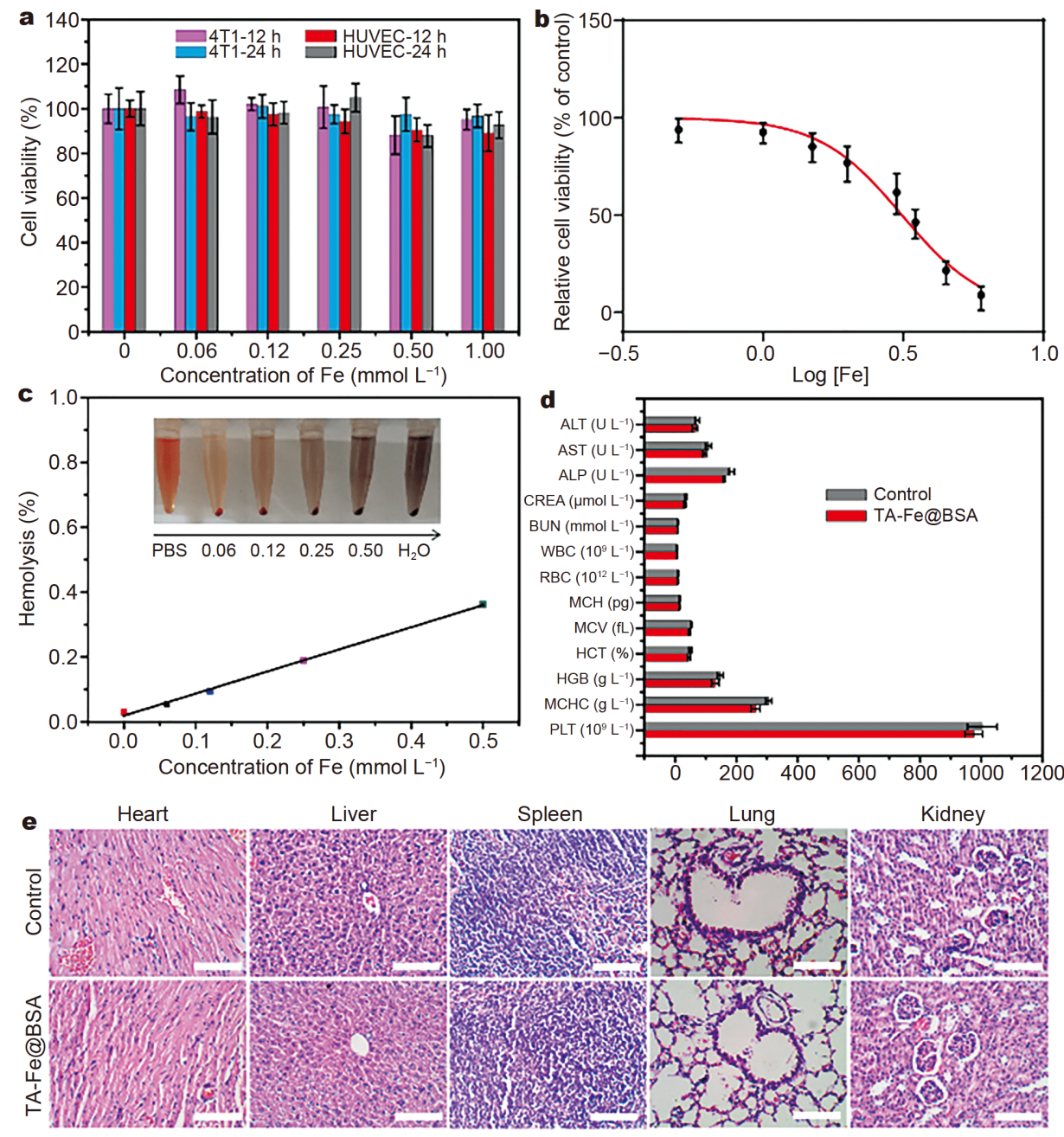

Figure 3 The biocompatibility assessment of TA-Fe@BSA. (a) The cell viability of HUVEC and 4T1 cells incubated with TA-Fe@BSA for 12 and 24 h, respectively. (b) IC50 dose response of TA-Fe@BSA. (c) Hemolysis percentage of RBCs after incubation with various concentrations of TA-Fe@BSA. $\mathrm{H}_{2} \mathrm{O}$ and PBS were used as positive and negative control, respectively. Inset shows photographs of the RBCs with different treatments. (d) Blood routine and biochemical indexes analyses for mice after intravenous injection of TA-Fe@BSA $(n=3)$. (e) H\&E stained slices of the heart, liver, spleen, lung and kidney of mice with and without intravenous injection of TA-Fe@BSA. Scale bar $=100 \mu \mathrm{m}$.

then decreased from 4 to $6 \mathrm{~h}$ (Fig. $5 \mathrm{c}$ and d). To determine the metabolic pathway, the biodistribution of TA-Fe@BSA in the major organs after intravenous administration was analyzed (Fig. S6). $\mathrm{Fe}^{3+}$ ions were found in the liver and spleen due to the uptake of NPs by the reticuloendothelial system (RES) organs $[38,39]$. Nevertheless, the accumulation of TA-Fe@BSA in the kidney was observed, consistent with previous reported serum albumin-assembly NPs $[40,41]$. The $\mathrm{Fe}^{3+}$ ions were present in the urinary and faecal excreta, suggesting TAFe@BSA can be cleared from the body. Therefore, we speculated that TA-Fe@BSA NPs were metabolized both in hepatic and renal pathway. In particular, the uptake of TA-Fe@BSA NPs in the tumor was approximately 12\%
$\mathrm{ID} \mathrm{g}^{-1}$ at $2 \mathrm{~h}$, and then gradually decreased, in accordance with MRI. This can be attributed to the enhanced permeability and retention (EPR) effect of NPs with a size range from 10 to $100 \mathrm{~nm}$, and polymers are especially suitable for the EPR effect [41-43]. In summary, TAFe@BSA with ultra-sensitive MR performance was demonstrated, and it can be exploited as an effective tumor MRI contrast agent for biomedical diagnosis.

As mentioned above, TA-Fe@BSA not only displayed enhanced MRI contrast, but also had obvious NIR absorption, which can be used for tumor treatment. Based on this characteristic, the photothermal properties of TAFe@BSA were characterized before being used for tumor PTT. Similar to the MRI results, photothermal imaging 

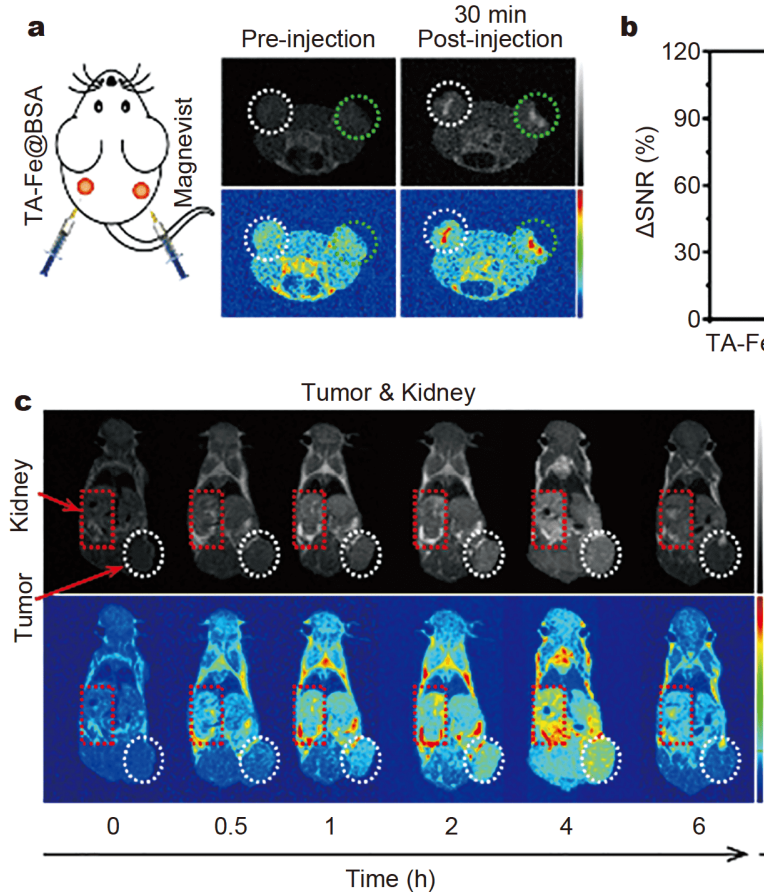

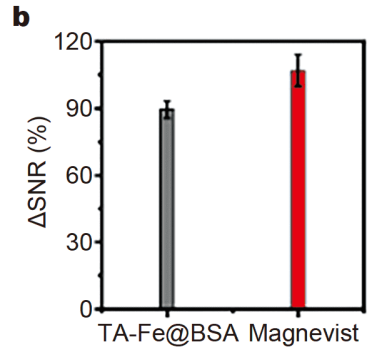

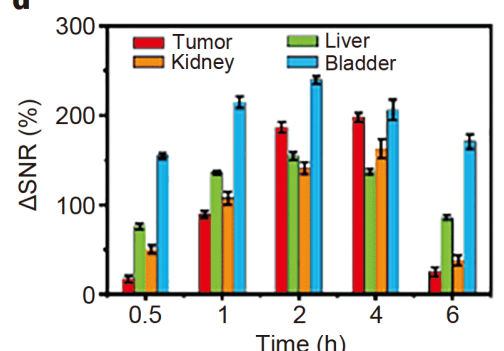

Liver \& Bladder

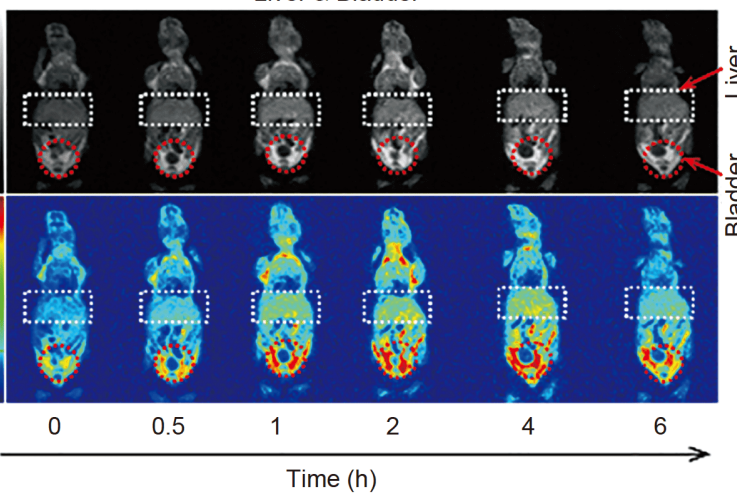

Figure 4 (a) $T_{1}$-weighted MR images (0.5 T) of bilateral tumor-bearing mice pre- and post-intratumor injection of TA-Fe@BSA (left) and Magnevist (right). (b) Corresponding signal changes of the pre- and post-injection in (a). The change of the signal to noise ratio ( $\Delta$ SNR) was calculated by $\left|\left(\mathrm{SNR}_{\text {post }}-\mathrm{SNR}_{\text {pre }}\right)\right| / \mathrm{SNR}_{\text {pre }}[36]$. (c) $T_{1}$-weighted MR images $(0.5 \mathrm{~T})$ of $4 \mathrm{~T} 1$ tumor-bearing mice after intravenous injection of TA-Fe@BSA. (d) The corresponding MR-signal variations of the tumor, liver, kidney and bladder in (c).

was also positively correlated with the concentration, and it was accompanied by increasing temperature (Fig. 6a and Fig. S7). The maximum temperature increased by $23^{\circ} \mathrm{C}$ in $15 \mathrm{~min}$, and the photothermal conversion efficiency was calculated to be $27.8 \%$ (Fig. $6 \mathrm{~b}$ ). In addition, the photostability was excellent after eight laser on/off cycles, and the absorption of the TA-Fe@BSA aqueous dispersion before and after laser irradiation remained virtually unchanged (Fig. $6 \mathrm{c}$ and Fig. S8). The relaxivities of TA-Fe@BSA did not change significantly before and after irradiation as well (Fig. S9). The above results demonstrate that TA-Fe@BSA can effectively convert laser energy into thermal energy, and it has the potential for use in PTT. To verify its feasibility, 4T1 cells incubated in a 96-well plate were randomly divided into four groups. They were then treated with either PBS, PBS+Laser, TAFe@BSA, or TA-Fe@BSA+Laser, and then stained with trypan-blue to evaluate the PTT effects. Only the cells of the TA-Fe@BSA+Laser irradiation group were stained blue, which indicated the safety of the laser and TAFe@BSA, as well as the good PTT effects (Fig. S10).

Given the excellent in vitro performance, tumor ablation via PTT was then further validated in vivo. For this,
4T1 tumor-bearing mice were randomly assigned to four groups. With MRI guidance, the strongest signal for the tumor was present in $\sim 2-4 \mathrm{~h}$. Therefore, for the treatment group, the mice were intravenously injected with TA-Fe@BSA, and then exposed to an $808 \mathrm{~nm}$ laser at $1 \mathrm{~W} \mathrm{~cm}^{-2}$ for $5 \mathrm{~min}$ at $2 \mathrm{~h}$ post-injection. The rest of the groups were used as controls, including PBS injection group, the group with mice that were irradiated after PBS injection, and the group with the mice injected with TAFe@BSA but without laser irradiation. Photothermal imaging of the mice tumor was carried out using an infrared imaging camera (Fig. 6d). Under $808 \mathrm{~nm}$ laser exposure, the tumor site of the TA-Fe@BSA+Laser group gradually brightened over time, accompanied by a significant temperature increase that was sufficient to ablate the tumor. In contrast, the tumor site in the PBS+Laser group barely changed and the temperature rise was lower (Fig. 6e). This demonstrated that TA-Fe@BSA had thermal ablation effects in vivo and can be used for PTT. Next, one mouse from each group was sacrificed and the tumors were excised for histopathological section analysis. Compared with the other three control groups, the largest necrotic area was observed in the TA-Fe@BSA 

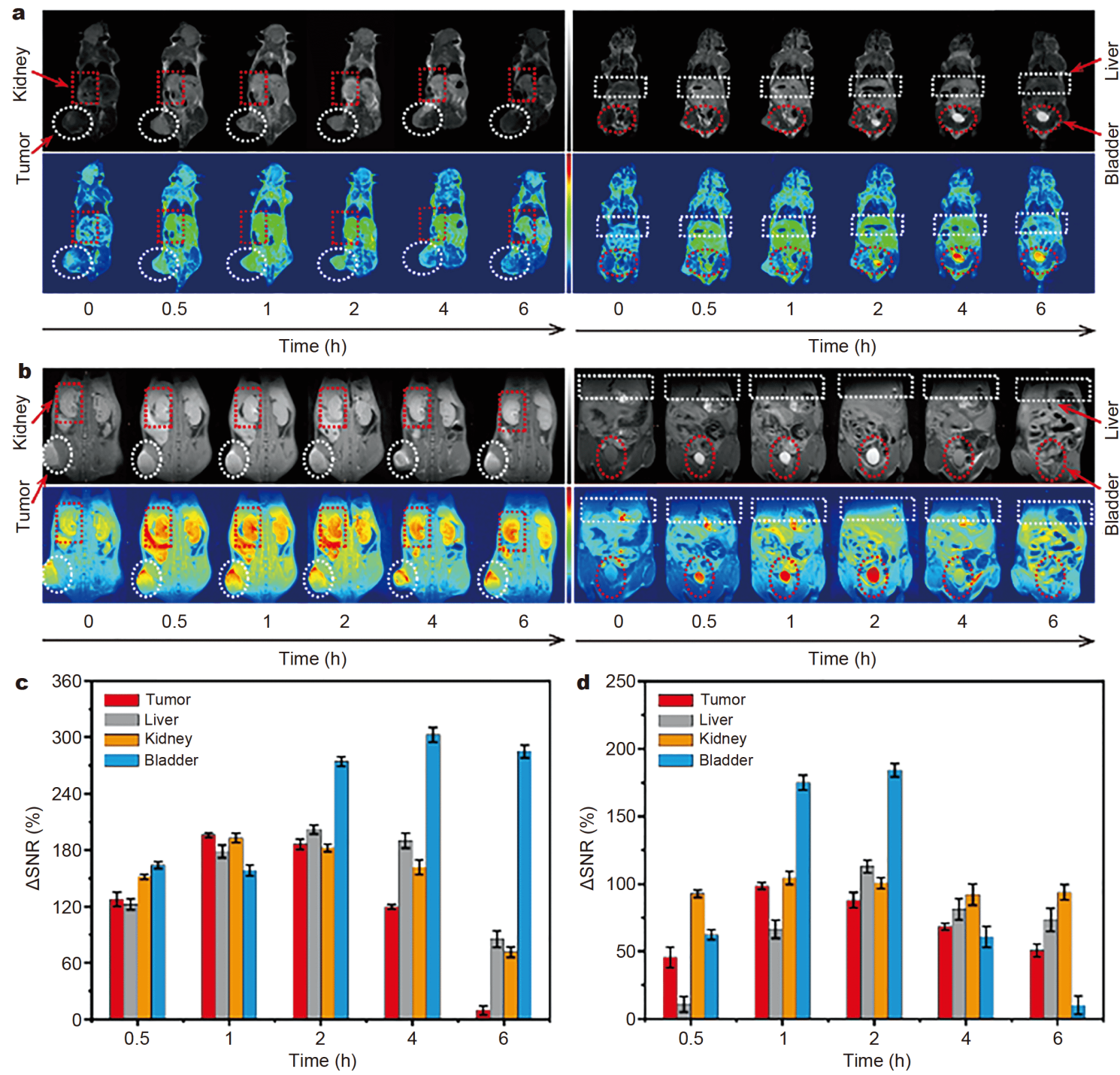

Figure 5 In vivo $T_{1}$-weighted MR images of $4 \mathrm{~T} 1$ tumor-bearing mice after intravenous injection of TA-Fe@BSA at (a) 1 and (b) 7 T, and the corresponding signal variations for the tumor, liver, kidney and bladder at (c) 1 and (d) 7 , respectively.

+Laser group from the $\mathrm{H} \& \mathrm{E}$ stained image. A large number of apoptosis cells were dyed brown in the TUNEL stained slices, where the TA-Fe@BSA+Laser group showed the highest apoptotic rate (Fig. $6 \mathrm{f}$ and g). Moreover, the tumor volume and body weight of the mice were monitored for 16 days. It can be clearly seen that the tumors of the treatment group were scabbed, which shrank remarkably and then completely disappeared 16 days after PPT treatment (Fig. S11). In contrast, the tumors in the other three groups were not suppressed, and neither laser irradiation nor TA-Fe@BSA alone affected tumor growth (Fig. 6h). In addition, all groups of mice maintained a steady weight over the entire PTT treat- ment, suggesting that TA-Fe@BSA had no significant toxic side effects, and therefore, has potential to be used as an effective PPT agent for tumor ablation.

\section{CONCLUSIONS}

In summary, the natural polyphenol TA, a ligand with a large and stable structure, was selected to construct TAFe@BSA as a $T_{1}$ MRI contrast agent and as a PTT agent for tumor theranostics. The obtained spherical TAFe@BSA had an average size of $\sim 21 \mathrm{~nm}$ and showed an obvious LMCT band and good biocompatibility. Compared with GA-Fe@BSA, it exhibited superior performance as an MRI contrast agent at 0.5, 1 and $7 \mathrm{~T}$ fields in 

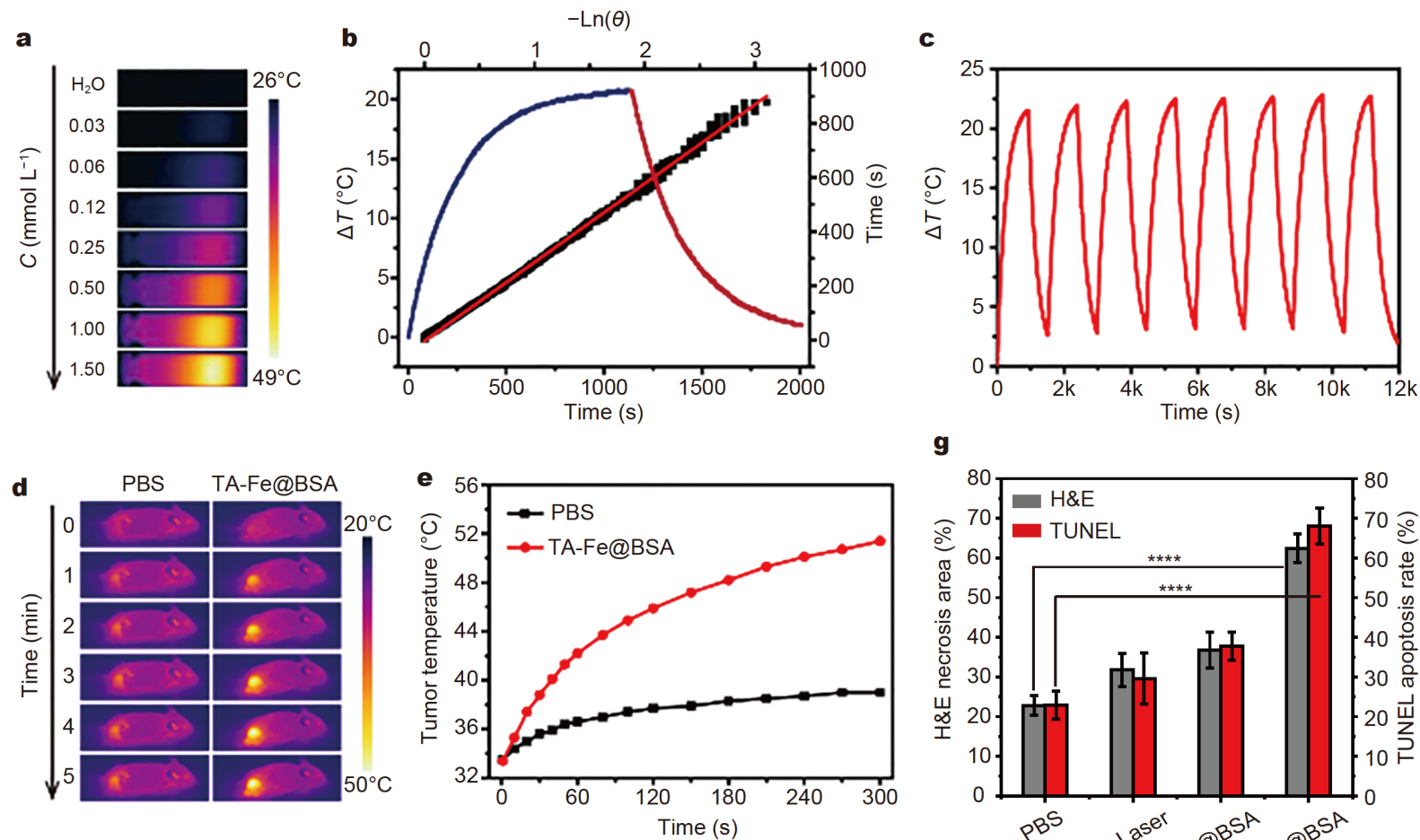

g
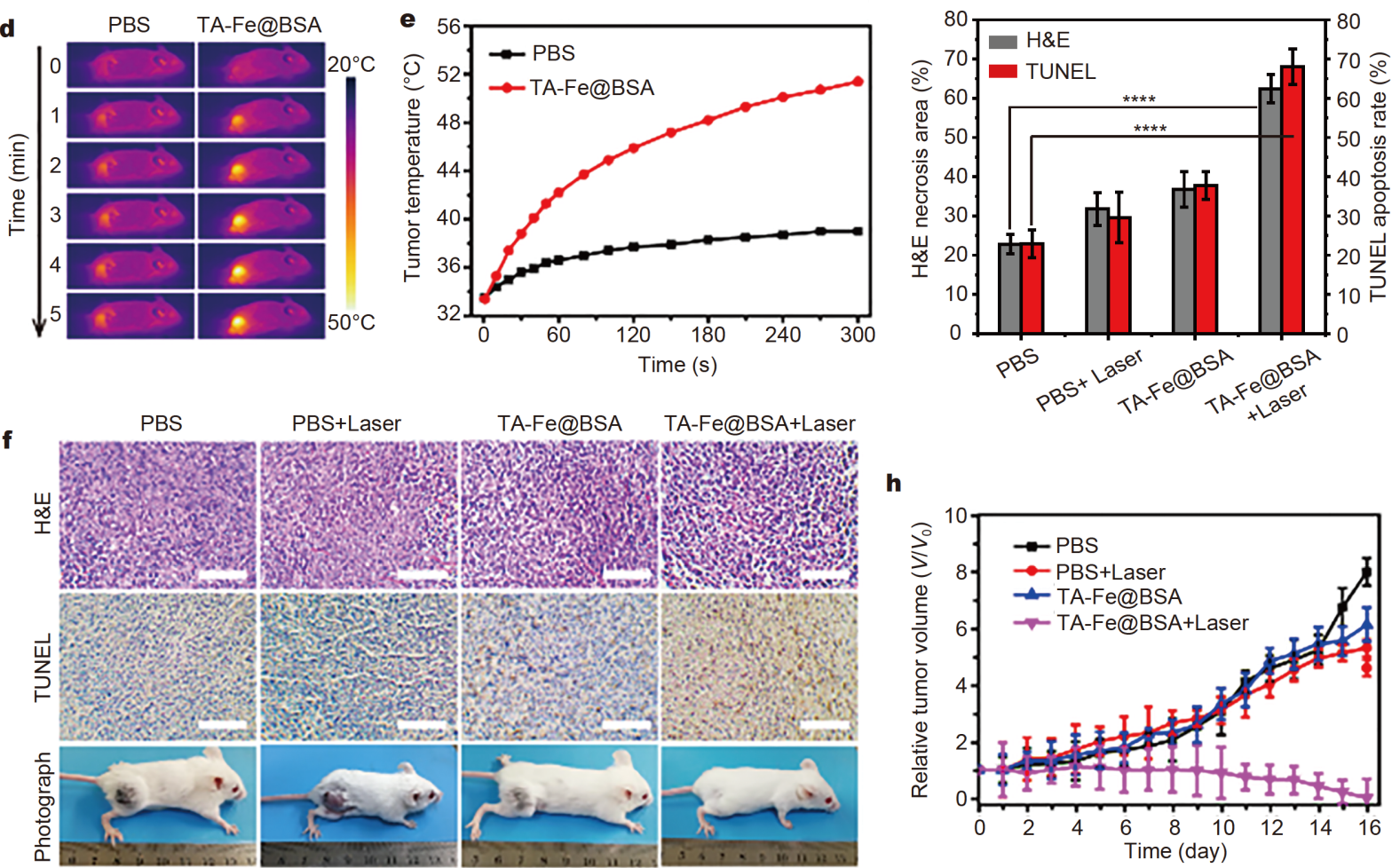

$\mathbf{h}$

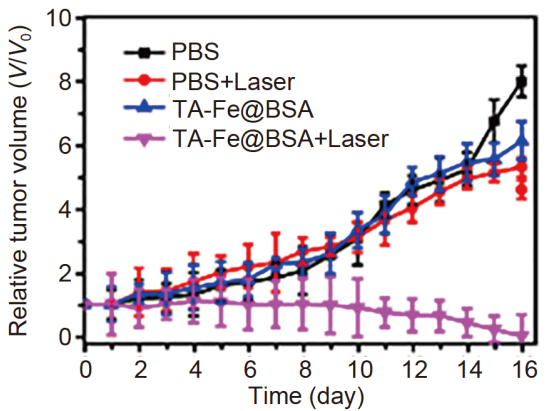

Figure 6 Evaluation of PTT performance and tumor ablation effects of TA-Fe@BSA. (a) NIR thermal images of TA-Fe@BSA with various Fe concentrations under irradiation at $808 \mathrm{~nm}\left(1 \mathrm{~W} \mathrm{~cm}^{-2}\right)$. (b) Heating and cooling rate curve of TA-Fe@BSA $\left(1 \mathrm{mmol} \mathrm{L}^{-1} \mathrm{Fe}\right)$ and fitting curve of time vs. $-\ln (\theta) .-\ln (\theta)$ was obtained from the cooling period. (c) Photothermal stability of TA-Fe@BSA over eight laser on/off cycles. (d) Thermal images of tumor for the PBS+Laser and TA-Fe@BSA+Laser groups under laser irradiation $\left(808 \mathrm{~nm}, 1 \mathrm{~W} \mathrm{~cm}^{-2}\right)$ for 5 min. (e) Corresponding temperature variation of (d). (f) H\&E, TUNEL staining slices (Scale bar $=100 \mu \mathrm{m}$ ) and representative photographs of tumors for different groups of mice. (g) Necrosis and apoptosis of tumor sections for different groups. (h) Relative tumor volume variation for different groups of mice $(n=5)$.

solution, and it demonstrated significantly improved relaxivity that was comparable to commercial Magnevist. The applicability of TA-Fe@BSA as a $T_{1}$-weighted MRI contrast agent was also validated in vivo after intratumoral and intravenous injection. Moreover, TAFe@BSA with NIR absorption presented efficient PTT effects for in vivo xenograft tumor suppression. This proof-of-concept study demonstrated that TA-Fe@BSA obtained via a simple synthesis route using large natural polyphenol ligands may be practical for theranostic applications.

Received 24 April 2020; accepted 20 June 2020; published online 4 September 2020

1 Logothetis NK. What we can do and what we cannot do with fMRI. Nature, 2008, 453: 869-878

2 Blow N. How to get ahead in imaging. Nature, 2009, 458: 926-928 3 Zheng XY, Zhao K, Tang J, et al. Gd-dots with strong ligand-water 
interaction for ultrasensitive magnetic resonance renography. ACS Nano, 2017, 11: 3642-3650

4 Sun C, Lin H, Gong X, et al. DOTA-branched organic frameworks as giant and potent metal chelators. J Am Chem Soc, 2020, 142: 198-206

5 Li H, Meade TJ. Molecular magnetic resonance imaging with Gd (III)-based contrast agents: Challenges and key advances. J Am Chem Soc, 2019, 141: 17025-17041

6 Que EL, New EJ, Chang CJ. A cell-permeable gadolinium contrast agent for magnetic resonance imaging of copper in a Menkes disease model. Chem Sci, 2012, 3: 1829-1834

$7 \mathrm{Hu} \mathrm{J}$, Liu S. Recent advances on stimuli-responsive macromolecular magnetic resonance imaging (MRI) contrast agents. Sci China Chem, 2018, 61: 1110-1122

$8 \mathrm{Kim} \mathrm{BH}$, Lee N, Kim H, et al. Large-scale synthesis of uniform and extremely small-sized iron oxide nanoparticles for high-resolution $T_{1}$ magnetic resonance imaging contrast agents. J Am Chem Soc, 2011, 133: 12624-12631

9 Wei H, Bruns OT, Kaul MG, et al. Exceedingly small iron oxide nanoparticles as positive MRI contrast agents. Proc Natl Acad Sci USA, 2017, 114: 2325-2330

10 Kanda T, Fukusato T, Matsuda M, et al. Gadolinium-based contrast agent accumulates in the brain even in subjects without severe renal dysfunction: Evaluation of autopsy brain specimens with inductively coupled plasma mass spectroscopy. Radiology, 2015, 276: 228-232

11 Le Fur M, Caravan P. The biological fate of gadolinium-based MRI contrast agents: A call to action for bioinorganic chemists. Metallomics, 2019, 11: 240-254

12 Sedó J, Saiz-Poseu J, Busqué F, et al. Catechol-based biomimetic functional materials. Adv Mater, 2013, 25: 653-701

13 Kuźnik N, Wyskocka M. Iron(III) contrast agent candidates for MRI: A survey of the structure-effect relationship in the last 15years of studies. Eur J Inorg Chem, 2016, 2016(4): 445-458

14 Snyder EM, Asik D, Abozeid SM, et al. A class of $\mathrm{Fe}^{\mathrm{III}}$ macrocyclic complexes with alcohol donor groups as effective $T_{1}$ MRI contrast agents. Angew Chem Int Ed, 2019, 59: 2414-2419

15 Wang H, Jordan VC, Ramsay IA, et al. Molecular magnetic resonance imaging using a redox-active iron complex. J Am Chem Soc, 2019, 141: 5916-5925

$16 \mathrm{Mu} \mathrm{X}$, Yan C, Tian Q, et al. BSA-assisted synthesis of ultrasmall gallic acid-Fe(III) coordination polymer nanoparticles for cancer theranostics. IJN, 2017, Volume 12: 7207-7223

17 Liu F, He X, Chen H, et al. Gram-scale synthesis of coordination polymer nanodots with renal clearance properties for cancer theranostic applications. Nat Commun, 2015, 6: 8003

18 Jun Y, Lee J, Cheon J. Chemical design of nanoparticle probes for high-performance magnetic resonance imaging. Angew Chem Int Ed, 2008, 47: 5122-5135

19 Zhou Z, Yang L, Gao J, et al. Structure-relaxivity relationships of magnetic nanoparticles for magnetic resonance imaging. Adv Mater, 2019, 31: 1804567

20 Kim BJ, Han S, Lee KB, et al. Biphasic supramolecular selfassembly of ferric ions and tannic acid across interfaces for nanofilm formation. Adv Mater, 2017, 29: 1700784

21 Wei J, Liang Y, Hu Y, et al. Hydrothermal synthesis of metalpolyphenol coordination crystals and their derived metal/N-doped carbon composites for oxygen electrocatalysis. Angew Chem Int Ed, 2016, 55: 12470-12474

22 Rahim MA, Ejima H, Cho KL, et al. Coordination-driven multistep assembly of metal-polyphenol films and capsules. Chem Mater, 2014, 26: 1645-1653

23 Ejima H, Richardson JJ, Liang K, et al. One-step assembly of coordination complexes for versatile film and particle engineering. Science, 2013, 341: 154-157

24 Caravan P. Strategies for increasing the sensitivity of gadolinium based MRI contrast agents. Chem Soc Rev, 2006, 35: 512-523

25 Marangoni VS, Neumann O, Henderson L, et al. Enhancing $T_{1}$ magnetic resonance imaging contrast with internalized gadolinium (III) in a multilayer nanoparticle. Proc Natl Acad Sci USA, 2017, 114: 6960-6965

26 Yang Z, Luo S, Zeng Y, et al. Albumin-mediated biomineralization of shape-controllable and biocompatible ceria nanomaterials. ACS Appl Mater Interfaces, 2017, 9: 6839-6848

27 Wang G, Siggers K, Zhang S, et al. Preparation of BMP-2 containing bovine serum albumin (BSA) nanoparticles stabilized by polymer coating. Pharm Res, 2008, 25: 2896-2909

28 Wen R, Lv X, Yang T, et al. Albumin nanoreactor-templated synthesis of $\mathrm{Gd}_{2} \mathrm{O}_{3} / \mathrm{CuS}$ hybrid nanodots for cancer theranostics. Sci China Mater, 2017, 60: 554-562

29 Wang L, Lin H, Ma L, et al. Albumin-based nanoparticles loaded with hydrophobic gadolinium chelates as $T_{1}-T_{2}$ dual-mode contrast agents for accurate liver tumor imaging. Nanoscale, 2017, 9: 4516-4523

30 Liu PY, Miao ZH, Li K, et al. Biocompatible $\mathrm{Fe}^{3+}$-TA coordination complex with high photothermal conversion efficiency for ablation of cancer cells. Colloids Surfs B-Biointerfaces, 2018, 167: 183-190

31 Wang $\mathrm{Y}, \mathrm{Wu} \mathrm{Y}$, Liu $\mathrm{Y}$, et al. BSA-mediated synthesis of bismuth sulfide nanotheranostic agents for tumor multimodal imaging and thermoradiotherapy. Adv Funct Mater, 2016, 26: 5335-5344

32 An L, Yan C, Mu X, et al. Paclitaxel-induced ultrasmall gallic acidFe@BSA self-assembly with enhanced MRI performance and tumor accumulation for cancer theranostics. ACS Appl Mater Interfaces, 2018, 10: 28483-28493

33 Avedano S, Tei L, Lombardi A, et al. Maximizing the relaxivity of HSA-bound gadolinium complexes by simultaneous optimization of rotation and water exchange. Chem Commun, 2007, 4726

34 Halle B. Molecular theory of field-dependent proton spin-lattice relaxation in tissue. Magn Reson Med, 2006, 56: 60-72

35 Bellin MF, Vasile M, Morel-Precetti S. Currently used non-specific extracellular MR contrast media. Eur Rad, 2003, 13: 2688-2698

36 Yang L, Wang Z, Ma L, et al. The roles of morphology on the relaxation rates of magnetic nanoparticles. ACS Nano, 2018, 12: 4605-4614

37 Ersoy H, Rybicki FJ. Biochemical safety profiles of gadoliniumbased extracellular contrast agents and nephrogenic systemic fibrosis. J Magn Reson Imag, 2007, 26: 1190-1197

38 Guerrero S, Herance JR, Rojas S, et al. Synthesis and in vivo evaluation of the biodistribution of a ${ }^{18} \mathrm{~F}$-labeled conjugate goldnanoparticle-peptide with potential biomedical application. Bioconjugate Chem, 2012, 23: 399-408

39 Zhang J, Hao G, Yao C, et al. Albumin-mediated biomineralization of paramagnetic NIR $\mathrm{Ag}_{2} \mathrm{~S}$ QDs for tiny tumor bimodal targeted imaging in vivo. ACS Appl Mater Interfaces, 2016, 8: 16612-16621

40 Chen Q, Feng L, Liu J, et al. Intelligent albumin- $\mathrm{MnO}_{2}$ nanoparticles as $\mathrm{pH}-/ \mathrm{H}_{2} \mathrm{O}_{2}$-responsive dissociable nanocarriers to modulate tumor hypoxia for effective combination therapy. Adv Mater, 2016, 28: 7129-7136

41 Sheng $\mathrm{Z}, \mathrm{Hu} \mathrm{D}$, Zheng $\mathrm{M}$, et al. Smart human serum albuminindocyanine green nanoparticles generated by programmed as- 
sembly for dual-modal imaging-guided cancer synergistic phototherapy. ACS Nano, 2014, 8: 12310-12322

42 Yang W, Guo W, Le W, et al. Albumin-bioinspired Gd:CuS nanotheranostic agent for in vivo photoacoustic/magnetic resonance imaging-guided tumor-targeted photothermal therapy. ACS Nano, 2016, 10: 10245-10257

43 Petros RA, DeSimone JM. Strategies in the design of nanoparticles for therapeutic applications. Nat Rev Drug Discov, 2010, 9: 615627

Acknowledgements This work was supported by the National Natural Science Foundation of China (91959105 and 21671135), Shanghai Sailing Program (19YF1436200), Shanghai Rising-Star Program (17QA1402600), Shanghai Talent Development Fund (2018082), and Shanghai Engineering Research Center of Green Energy Chemical Engineering (18DZ2254200).

Author contributions An L analyzed the data and wrote the article; Cai Y carried out the experiments; Lin J participated in the discussion of the manuscript; Yang S and Tian Q supervised the project and were responsible for writing, editing and data curation. All authors contributed to the general discussion.

Conflict of interest The authors declare that they have no conflict of interest.

Supplementary information online version of the paper.

Supporting data are available in the

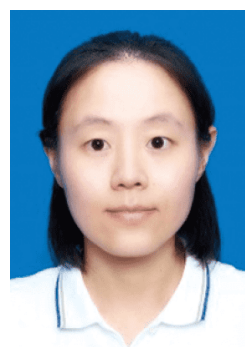

Lu An received her Master degree from Shanghai Normal University in 2012. She is currently a $\mathrm{PhD}$ candidate under the supervision of Prof. Shiping Yang. Her research interest focuses on the development of multimodal imaging probes for theranostic applications.

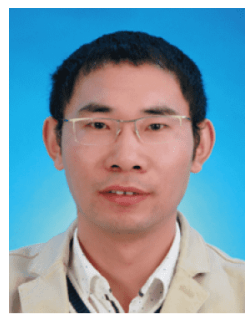

Qiwei Tian obtained his $\mathrm{PhD}$ degree in materials science from Donghua University, China in 2012, and worked as a Postdoctoral Fellow at King Abdullah University of Science and Technology in 2012-2015. He is currently working as an associate professor at Shanghai Normal University and his research is focused on the development of smart agents for cancer theranostics.

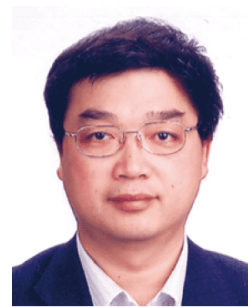

Shiping Yang is a professor of the Key Laboratory of Resource Chemistry of Ministry of Education, Shanghai Normal University. He received his $\mathrm{PhD}$ degree in Sun Yat-sen University in 2000. His research interest is in the field of biomedical applications of nanomaterials for novel biomedical imaging and cancer therapy.

\section{天然多酚单宁酸构建的铁基超灵敏磁共振造影剂 及其肿瘤诊疗应用}

安璐, 蔡宇, 田启威, 林焦敏, 杨仕平 ${ }^{*}$

摘要 $\mathrm{Gd}(\mathrm{III})$ 配合物和超顺磁性氧化铁 (SPION) 是目前常见的 $T_{1}$ MRI造影剂, 然而由于 Gd(III)的毒性和SPION较差的对比度, 需要 开发出新的稳定、无毒的高效造影剂. 本论文基于减慢分子自旋 的策略, 利用生物安全的天然多酚单宁酸和牛血清白蛋白来构建 无毒的 Fe (III)复合物 TA-Fe@BSA. 该复合物具有良好的增强弛豫 性能, 在溶液和体内测试中, 在 $0.5,1$ 和 $7 \mathrm{~T}$ 磁场下均具有与商用 $T_{1}$ 造影剂Magnevist ${ }^{\mathbb{B}}$ 相当的 $T_{1}$ MRI增强造影效果, 而且具有良好的 稳定性和生物相容性. 此外, 具有近红外吸收的TA-Fe@BSA基于其 光热效应, 也表现出了有效的肿瘤消融作用. 这些结果证明了TAFe@BSA 作为替代性 $T_{1}$ MRI造影剂和肿瘤治疗药物的潜力. 